\title{
Potent Antiretroviral Therapy
}

National Cancer Institute

\section{Source}

National Cancer Institute. Potent Antiretroviral Therapy. NCI Thesaurus. Code C157520.

Antiretroviral therapy using two or more nucleoside reverse transcriptase inhibitors with either a protease inhibitor or a non-nucleoside reverse transcriptase inhibitor. 